NASA Technical Memorandum 103627

AIAA-90-3954

\title{
Prediction of the Noise From a Propeller at Angle of Attack
}

Eugene A. Krejsa

Lewis Research Center

Cleveland, Ohio

Prepared for the

13th Aeroacoustics Conference

sponsored by the American Institute of Aeronautics and Astronautics

Tallahassee, Florida, October 22-24, 1990

\section{NASn}




\title{
PREDICTION OF THE NOISE FROM A PROPELLER
}

\author{
AT ANGLE OF ATTACK \\ Eugene A. Krejsa \\ National Aeronautics and Space Administration \\ Lewis Research Center \\ Cleveland, Ohio 44135
}

\begin{abstract}
SUMMARY
An analysis is presented to predict the noise of a propeller at angle of attack. The analysis is an extension of that reported by Mani in NASA CR-4264 which predicted the change in noise due to angle of attack due to both unsteady loading and to azimuthal variation of the radiation efficiency of steady noise sources. Mani's analysis, however, was limited to small angles of attack. The analysis reported herein removes this small angle limitation. Results from the analysis are compared with the data of Woodward, NASA TM-88920, for a single rotation propeller and, TM-100206, for a counter rotating propeller. The comparison shows that including the effect of angle of attack on the steady noise sources significantly improves the agreement with data. Including higher order effects of angle of attack, while changing the predicted noise at far forward and aft angles, has little effect near the propeller plane.
\end{abstract}

\section{INTRODUCTION}

Advanced turboprop powered aircraft have the potential of significantly reducing the fuel consumed by commercial aircraft. A model of a typical single rotation advanced propeller is shown in figure 1. For aircraft powered by this type of propeller to be viable concepts, these aircraft must meet noise rules, both international, national, i.e., FAR-36, and local. Much research has been conducted on the noise from many variations of advanced turboprop designs. Most of this research has been conducted with the propeller axis parallel to the flow direction. However, results by Woodward (refs. 1 and 2), Woodward and Gordon (ref. 3) and Block (refs. 4 and 5) have shown that the noise of a propeller at angle of attack is significantly different than that of a propeller at zero angle of attack.

Like the experimental work, most of the analysis of propeller noise has been directed to the noise from propellers without angle of attack. When a propeller is at angle of attack relative to the flow direction, the loading on the propeller varies with azimuthal angle. Several authors (refs. 6 to 9) have included the effect of this unsteady loading in their analyses. However, when applying this type of analysis to recent data from propellers at angle of attack, the authors of reference 9 found that the analysis predicted the effects of angle of attack for lightly loaded propellers but underpredicted the effect for highly loaded propellers. Mani (ref. 10) has recently presented an analysis that, in addition to unsteady loading, also includes the effect of angle of attack on the vadiation of the steady loading and thickness noise sources. An earlier paper by Stuff (ref. 11) also included this effect, but only for a rotating point source. Mani's analysis allows the source to be distributed radially. A report by Padula and Block (ref. 12) also recognized this effect in their application of Farassat's time domain analysis of propeller noise. Mani's analysis was done in the frequency domain, where the mechanisms by which angle of attack alters the noise production and radiation appear 
explicitly and are more easily identified. However, Mani's analysis is limited to first order effects of angle of attack.

The analysis presented in this paper is also done in the frequency domain and follows the derivation of Parry (ref. 13) with modification to account for angle of attack. No assumptions regarding the magnitude of the angle of attack are made. Results of the analysis are compared with data of references 1 and 2 for the SR7A single rotation propeller and the F7A7 counterrotation propeller, respectively. Both of these propellers are highly loaded and were tested over a range of angles of attack at simulated approach conditions.

\section{ANALYSIS}

The problem is formulated in a coordinate system with its $x$ axis (fig. 2) aligned with the mean flow direction. (This differs from Mani's analysis in which the coordinate system was aligned with the propeller axis.) In the coordinate system of the present analysis, the source moves in a plane which is at an angle, equal to the angle of attack, relative to the $y-z$ plane. Thus the source has periodic axial motion in addition to its circular motion. Likewise, when the blade loading is resolved into components in the current coordinate system, a component of thrust becomes a vertical force component. It is these added effects that, in addition to the unsteady loading, give rise to additional noise terms due to angle of attack.

\section{Loading Noise}

The noise generated due to a point force is given by Parry as:

$$
\mathrm{dP}_{\mathrm{F}}=-\nabla \cdot\left[\frac{\mathrm{F}(\tau)}{4 \pi \mathrm{R}(1-\mathrm{M})}\right]
$$

where $\tau$ is the retarded time given by:

$$
\tau=\frac{t-\frac{R}{C_{0}}}{1-M_{x} \cos \theta}
$$

and $F(\tau)$ is the force per unit of blade area evaluated at the retarded time $\tau$. $F(\tau)$ is given by:

$$
F=\frac{1}{2} \rho U_{r}^{2} C_{L} \frac{c B}{2 \pi} \sum_{k=-\infty}^{\infty} F_{k} \exp \left\{i\left[( \pm m B+k) \phi-\frac{m B \Omega\left(t-\frac{R}{C_{0}}\right)}{1-M_{x} \cos \theta}\right]\right\}
$$

where the + sign refers to counter-clockwise, forward-looking aft (FLA), propeller rotation and the - sign refers to clockwise rotation. The term 
$M_{x} \cos \theta$ is the component of the propeller hub motion in the direction of the observer. All symbols are defined in the appendix.

This formulation differs from Parry in two respects. First, the sign convention for the time dependence has been changed to be consistant with Mani.

And second, the force has been assumed to vary circumferentially and is

expressed as a sum of circumferential modes, with each mode having an amplitude given by $\mathrm{C}_{\mathrm{L}} \mathrm{F}_{\mathrm{k}}$. In general, $\mathrm{F}_{\mathrm{k}}$ can be complex to allow for the maximum loading of each mode to occur at different azimuthal locations. From (1) and (2), the far field pressure due to a point force is:

$\mathrm{dP}_{\mathrm{F}}=-\frac{\mathrm{imB} \Omega}{4 \pi \mathrm{R}_{\mathrm{O}} \mathrm{C}_{\mathrm{o}}\left(1-\mathrm{M}_{\mathrm{X}} \cos \theta\right)^{2}}\left(\cos \theta \mathrm{F}_{\mathrm{X}}+\cos \phi_{\mathrm{o}} \sin \theta \mathrm{F}_{\mathrm{y}}+\sin \phi_{\mathrm{o}} \sin \theta \mathrm{F}_{\mathrm{z}}\right)$

where for a propeller at angle of attack, $\beta$, the components of $F$ (See figs. 2 and 3 for schematics of the source and observer coordinates.) are given by:

$$
\begin{aligned}
& \mathrm{F}_{\mathrm{x}}=[\sin \alpha \cos \beta+\cos \alpha \cos (\phi+\psi) \sin \beta] \mathrm{F} \\
& \mathrm{F}_{\mathrm{y}}=\cos \alpha \sin (\phi+\psi) \mathrm{F} \\
& \mathrm{F}_{\mathrm{z}}=-[\cos \alpha \cos (\phi+\psi) \cos \beta-\sin \alpha \sin \beta] \mathrm{F}
\end{aligned}
$$

where

$$
\alpha=\tan ^{-1}\left(\frac{\frac{r}{r_{t}} M_{T}}{M_{x}}\right)
$$

The distance between the observer location and the source location, $R$, is given by:

$$
R=\sqrt{\left(x_{0}-x_{s}\right)^{2}+\left(y_{0}-y_{s}\right)^{2}+\left(z_{0}-z_{s}\right)^{2}}
$$

where $x_{0}, y_{0}$, and $z_{0}$ are the observer coordinates and $x_{S}, y_{S}$, and $z_{S}$ are the source coordinates.

For a propeller at angle of attack, the source coordinates are:

$$
\left.\begin{array}{l}
x_{S}=-(\Delta X-c X \cos \alpha) \cos \beta-r \sin (\phi+\psi) \sin \beta \\
y_{S}=r \cos (\phi+\psi) \\
z_{S}=-(\Delta X-c X \cos \alpha) \sin \beta+r \sin (\phi+\psi) \cos \beta
\end{array}\right\}
$$

with

$$
\psi=\psi_{0}+\frac{c X}{r} \sin \alpha
$$


In the far-field,

$$
\begin{aligned}
\mathrm{R} \simeq \mathrm{R}_{\mathrm{o}}+\cos \theta[ & (\Delta \mathrm{X}-\mathrm{CX} \cos \alpha) \cos \beta+\mathrm{r} \sin (\phi+\psi) \sin \beta] \\
& -\cos \phi_{0} \sin \theta \mathrm{r} \cos (\phi+\psi) \\
& -\sin \phi_{0} \sin \theta[-(\Delta X-\mathrm{cX} \cos \alpha) \sin \beta+\mathrm{r} \sin (\phi+\psi) \cos \beta]
\end{aligned}
$$

Combining equations (4), (5), and (9) and integrating over the source region, with the source concentrated at $X=0$, gives:

$$
\begin{aligned}
& P_{F}=-\sum_{m=-\infty}^{\infty} \frac{i m B^{2} \Omega_{\rho_{0} c_{0}}}{16 \pi^{2} R_{0}\left(1-M_{x} \cos \theta\right)^{2}} \exp \left[-\frac{i m B \Omega\left(t-\frac{R_{0}}{c_{0}}\right)}{1-M_{x} \cos \theta}\right]_{\text {Hub }}^{T i p} \mathrm{cM}_{r}^{2} C_{L} \sum_{k=-\infty}^{\infty} F_{k} \\
& x \exp \left[\frac{i m B \Omega}{C_{0}\left(1-M_{x} \cos \theta\right)}\left(\cos \theta \cos \beta+\sin \theta \sin \phi_{0} \sin \beta\right) \Delta X\right] \\
& \times \int_{0}^{2 \pi}\left[\sin \alpha\left(\cos \theta \cos \beta+\sin \theta \sin \phi_{0} \sin \beta\right)+\cos \alpha(\cos \theta \sin \beta\right. \\
& \left.\left.-\sin \theta \sin \phi_{0} \cos \beta\right) \cos \left(\phi+\psi_{0}\right)+\cos \alpha \sin \theta \cos \phi_{0} \sin \left(\phi+\psi_{0}\right)\right] \\
& x \exp \left(i \left\{( \pm m B+k) \phi-\frac{m B \Omega}{C_{0}\left(1-M_{x} \cos \theta\right)}\left[-r \cos \phi_{0} \sin \theta \cos \left(\phi+\psi_{0}\right)\right.\right.\right. \\
& \left.\left.\left.+r\left(\cos \theta \sin \beta-\sin \theta \sin \phi_{0} \cos \beta\right) \sin \left(\phi+\psi_{0}\right)\right]\right\}\right) d \phi d r
\end{aligned}
$$

The integral over $\phi$ can be written as:

$$
\begin{aligned}
\Phi=\int_{0}^{2 \pi}\{ & \sin \alpha\left(\cos \theta \cos \beta+\sin \theta \sin \phi_{0} \sin \beta\right)+\cos \alpha[\cos \theta \sin \beta \\
& \left.\left.+\sin \theta \sin \phi_{0}(1-\cos \beta)\right] \cos \left(\phi+\psi_{0}\right)+\cos \alpha \sin \theta \sin \left(\phi-\phi_{0}+\psi_{0}\right)\right\} \\
& \times \exp \left[i \left(( \pm m B+k) \phi+\frac{m B \Omega r}{c_{0}\left(1-M_{x} \cos \theta\right)}\left\{-\sin \theta \cos \left(\phi-\phi_{0}+\psi_{0}\right)\right.\right.\right. \\
& \left.\left.\left.+\left[\cos \theta \sin \beta+\sin \theta \sin \phi_{0}(1-\cos \beta)\right] \sin \left(\phi+\psi_{0}\right)\right\}\right)\right] d \phi
\end{aligned}
$$


Equation (11) is of the form:

$$
\begin{aligned}
\Phi= & \int_{0}^{2 \pi}\left[A_{1}+A_{2} \cos \left(\phi+\psi_{0}\right)+A_{3} \sin \left(\phi-\phi_{0}+\psi_{0}\right)\right] \\
& \quad x \exp [i( \pm m B+k) \phi] \exp \left[-i z_{1} \cos \left(\phi-\phi_{0}+\psi\right)\right] \exp \left[i z_{2} \sin \left(\phi+\psi_{0}\right)\right] d \phi
\end{aligned}
$$

where

$$
\begin{aligned}
& A_{1}=\sin \alpha\left(\cos \theta \cos \beta+\sin \theta \sin \phi_{0} \sin \beta\right) \\
& A_{2}=\cos \alpha\left[\cos \theta \sin \beta+\sin \theta \sin \phi_{0}(1-\cos \beta)\right] \\
& A_{3}=\cos \alpha \sin \theta
\end{aligned}
$$

and

$$
\begin{aligned}
& z_{1}=\frac{m B \Omega r \sin \theta}{C_{0}\left(1-M_{x} \cos \theta\right)} \\
& z_{2}=\frac{z_{1}}{\sin \theta}\left[\cos \theta \sin \beta+\sin \theta \sin \phi_{0}(1-\cos \beta)\right]
\end{aligned}
$$

Using the Bessel generating function (ref. 14):

$$
\exp \left[-i z_{1} \cos \left(\phi-\phi_{0}+\psi_{0}\right)\right]=\sum_{n_{1}=-\infty}^{\infty} J_{n_{1}}\left(z_{1}\right) \exp \left[-i n_{1}\left(\phi-\phi_{0}+\psi_{0}+\frac{\pi}{2}\right)\right]
$$

and

$$
\exp \left[i z_{2} \sin \left(\phi+\psi_{0}\right)\right]=\sum_{n=-\infty}^{\infty} J_{n}\left(z_{2}\right) \exp \left[i n_{2}\left(\phi+\psi_{0}\right)\right]
$$

Combining (12) and (15) yields:

$$
\begin{aligned}
& \Phi=\int_{0}^{2 \pi}\left[A_{1}+A_{2} \cos (\phi+\psi)+A_{3}\left(\phi-\phi_{0}+\psi_{0}\right)\right] \\
& \times \sum_{n_{1}=-\infty}^{\infty} \sum_{n_{2}=-\infty}^{\infty} J_{n_{1}}\left(z_{1}\right) J_{n_{2}}\left(z_{2}\right) \exp \left\{i \left[\left( \pm m B+k-n_{1}+n_{2}\right) \phi+n_{1}\left(\phi_{0}-\frac{\pi}{2}\right)\right.\right. \\
&\left.\left.-\left(n_{1}-n_{2}\right) \psi_{0}\right]\right\} d \phi
\end{aligned}
$$


which when integrated gives:

$$
\begin{aligned}
\Phi=2 \pi \exp \left[-i( \pm m B+k)\left(\psi_{0}+\frac{\pi}{2}-\phi_{0}\right)\right] \sum_{n_{2}=-\infty}^{\infty} J_{ \pm m B+k+n_{2}}\left(z_{1}\right) \\
\times J_{n_{2}}\left(z_{2}\right) \exp \left\{i n_{2}\left(\phi_{0}-\frac{\pi}{2}\right)\left[A_{1}+\frac{n_{2} A_{2}}{z_{2}}-\frac{\left( \pm m B+k+n_{2}\right)}{z_{1}} A_{3}\right]\right\}
\end{aligned}
$$

Combining (10), (13), and (17)

$$
\begin{aligned}
&\left.P_{F}=\sum_{m=-\infty}^{\infty}-\frac{i m B^{2} \Omega \rho_{0} C_{0}}{8 \pi R_{0}(1-}-M_{x} \cos \theta\right)^{2} \exp \left\{-\left[\frac{i m B \Omega\left(t-\frac{R_{0}}{C_{0}}\right)}{1-M_{x} \cos \theta}\right]\right\} \int_{H u b}^{T i p} c_{r}^{2} C_{L} \sum_{k=-\infty}^{\infty} F_{k} \\
& \times \exp \left[\frac{i m B \Omega}{1-M_{x} \cos \theta}\left(\cos \theta \cos \beta+\sin \theta \sin \Phi_{0} \sin \beta\right) \Delta x\right] \\
& \times \exp \left[-i( \pm m B+k)\left(\psi_{0}+\frac{\pi}{2}-\phi_{0}\right)\right] \sum_{n_{2}=-\infty}^{\infty} J_{ \pm m B+k+n_{2}}\left(z_{1}\right) J_{n_{2}}\left(z_{2}\right) \\
& \times \exp \left[i n_{2}\left(\phi_{0}-\frac{\pi}{2}\right)\right]\left[\sin \alpha\left(\cos \theta \cos \beta+\sin \theta \sin \phi_{0} \sin \beta\right)\right. \\
&\left.-\frac{( \pm m B+k) \cos \alpha\left(1-M_{x} \cos \theta\right)}{\frac{m B \Omega r}{C_{0}}}\right] d r
\end{aligned}
$$

Equation (18) gives the total far-field noise due to the blade loading. Both the change in loading with angle of attack and angle of attack effects on the steady loading noise are included. The relative importance of the terms in equation (18) will be discussed later.

\section{Thickness Noise}

The noise generated by a point volume source is given in reference 10 as:

$$
d P_{V}=\frac{\partial}{\partial t}\left[\frac{\rho V_{n}}{4 \pi(1-M) R}\right] d S
$$


where $V_{n}$ is the fluid velocity normal to the blade surface and is given by:

$$
V_{n}=c_{o} \frac{b}{c} M_{r} \frac{\partial h}{\partial X} \frac{B}{2 \pi} \sum_{m=-\infty}^{\infty} \exp [\operatorname{imB}( \pm \phi-\Omega \tau)]
$$

With angle of attack, $M_{r}$ will vary azimuthally and this variation will be expressed as a sum of azimuthal modes of magnitude $\mathrm{M}_{\mathrm{rk}}$. In general $\mathrm{M}_{\mathrm{rk}}$ will be complex to allow for phasing of the modes. Thus,

$$
M_{r}=\sum_{k=-\infty}^{\infty} M_{r k} \exp (i k \phi)
$$

Combining (20) and (21) and using equation (2) gives:

$$
V_{n}=c_{0} \frac{b}{c} \frac{\partial h}{\partial X} \frac{B}{2 \pi} \sum_{m=-\infty}^{\infty} \sum_{k=-\infty}^{\infty} M_{r k} \exp \left\{i\left[( \pm m B+k) \phi-\frac{m B \Omega\left(t-\frac{R}{c_{0}}\right)}{1-M_{x} \cos \theta}\right]\right\}
$$

Substituting (22) into (19) and integrating over the source region gives:

$$
\begin{aligned}
& P_{V}=\int_{\text {Hub }}^{\text {Tip }} \int_{0}^{2 \pi} \int_{\text {Leading edge }}^{\text {Trail ling edge }} \frac{\rho_{0} c_{0} \frac{b}{c} \frac{\partial h(X)}{\partial X} B}{8 \pi^{2}\left(1-M_{x} \cos \theta\right) R_{0}} \sum_{m=-\infty}^{\infty}-\frac{i m B \Omega}{1-M_{x} \cos \theta} \\
& x \exp \left\{i\left[( \pm m B+k) \phi-\frac{m B \Omega\left(t-\frac{R}{C_{0}}\right)}{1-M_{x} \cos \theta}\right] d x d \phi d r\right.
\end{aligned}
$$


Using (9) gives:

$$
\begin{aligned}
& P_{V}=-\frac{i \rho_{0} C_{0} B \Omega}{8 \pi^{2}\left(1-M_{x} \cos \theta\right)^{2}} \sum_{m=-\infty}^{m B} \exp \left[-\frac{i m B \Omega\left(t-\frac{R_{0}}{C_{0}}\right)}{1-M_{x} \cos \theta} \int_{H u b}^{T i p} \frac{b}{C} \sum_{k=-\infty}^{\infty} M_{r k}\right. \\
& \times \int_{-y_{2}}^{\infty} \frac{\partial h}{\partial X} \exp \left[\frac{i m B \Omega}{C_{0}\left(1-M_{x} \cos \theta\right)}\left(\cos \theta \cos \beta+\sin \theta \sin \phi_{0} \sin \beta\right)(\Delta X-\cos \alpha)\right] \\
& \times \int_{0}^{2 \pi} \exp \left(i \left\{( \pm m B+k) \phi+\frac{m B \Omega}{C_{0}\left(1-M_{x} \cos \theta\right)}\left[-r \cos \phi_{0} \sin \theta \cos (\phi+\psi)\right.\right.\right. \\
& \left.\left.\left.+r\left(\cos \theta \sin \beta-\sin \theta \sin \phi_{0} \cos \beta\right) \sin (\phi+\psi)\right]\right\}\right) d \phi d x d r
\end{aligned}
$$

Evaluating the $\phi$ integral in a manner similar to that used for the loading noise gives:

$$
\begin{aligned}
& P_{V}=-\frac{i \rho_{0} c_{0} B \Omega}{4 \pi\left(1-M_{x} \cos \theta\right)^{2} R_{0}} \sum_{m=-\infty}^{\infty} m \exp \left[-\frac{i m B \Omega\left(t-\frac{R_{0}}{c_{0}}\right)}{1-M_{x} \cos \theta} \int_{\text {Hub }}^{T i p} \underline{b} \sum_{k=-\infty}^{\infty} M_{r k}\right. \\
& \times \int_{-y_{2}}^{y_{2}} \frac{\partial h}{\partial X} \exp \left[\frac{i m B \Omega}{c_{0}\left(1-M_{x} \cos \theta\right)}\left(\cos \theta \cos \beta+\sin \theta \sin \phi_{0} \sin \beta\right)(\Delta X-\operatorname{cX} \cos \alpha)\right] \\
& \times \exp \left[-i( \pm m B+k)\left(\psi_{0}+\frac{c X}{r} \sin \alpha+\frac{\pi}{2}-\phi_{0}\right)\right] \\
& x \sum_{n_{2}=-\infty}^{\infty} J_{ \pm m B+k+n_{2}}\left(z_{1}\right) J_{n_{2}}\left(z_{2}\right) \exp \left[i n_{2}\left(\phi_{0}-\frac{\pi}{2}\right)\right] d x d r
\end{aligned}
$$


Evaluating the $\mathrm{x}$ integral by parts and concentrating the blade thickness at the mid-chord yields:

$$
\begin{aligned}
P_{V}= & \sum_{m=-\infty}^{\infty} \frac{m B^{2} \Omega p_{0} C_{C}}{4 \pi R_{0}\left(1-M_{x} \cos \theta\right)^{2}} \exp \left[-\frac{i m B \Omega\left(t-\frac{R_{0}}{C_{0}}\right)}{1-M_{x} \cos \theta}\right] \\
& \times \int_{H u b}^{T} \frac{b}{c} \sum_{k=-\infty}^{\infty i p} M_{r k} \exp \left[\frac{i m B \Omega}{1-M_{x} \cos \theta}\left(\cos \theta \cos \beta+\sin \theta \sin \phi_{0} \sin \beta\right) \Delta x\right] \\
& \times \exp \left[-i( \pm m B+k)\left(\psi_{0}+\frac{\pi}{2}-\phi_{0}\right)\right] \sum_{n_{2}=-\infty}^{\infty} J_{ \pm m B+k+n_{2}}\left(z_{1}\right) J_{n_{2}}\left(z_{2}\right) \\
& \times \exp \left[i n_{2}\left(\phi_{0}-\frac{\pi}{2}\right)\right]\left[\frac{m B \Omega c}{c_{0}\left(1-M_{x} \cos \theta\right)}\right. \\
& \left.\times\left(\cos \theta \cos \beta+\sin \theta \sin \phi_{0} \sin \beta\right)+( \pm m B+k) \frac{c}{r} \sin \alpha\right]
\end{aligned}
$$

Equation (26) gives the noise due to blade thickness. Like the result for loading noise, both steady and angle of attack effects are included.

\section{DISCUSSION OF RESULTS}

Equations (18) and (26) give the far-field noise for loading and thickness noise respectively. The two equations contain similar terms accounting for the various effects of angle of attack. The interpretation of these terms is the same for both sources. Since the results will be compared with data for conditions where the loading noise prevails, only the result for loading noise will be discussed.

\section{Discussion of Analytical Results}

Equation (18) gives the far-field noise due to both steady, $\mathrm{k}=0$, and unsteady loading, $k \neq 0$, and includes the effects of periodic axial source motion and variation in force direction. The unsteady loading, axial source motion, and variation in force direction are all due to angle of attack.

The noise due to unsteady loading depends directly on the magnitude of $\mathrm{C}_{\mathrm{L}} \mathrm{F}_{\mathrm{k}}$, the circumferential mode amplitude. However, as pointed out by other authors (refs. 6 to 9), values of $k$ that reduce the order of $J_{ \pm m B+k+n_{2}}$, will 
radiate more efficiently than those that increase the order. Thus for a counter-clockwise (FLA) rotating propeller $(+\mathrm{mB})$ the -1 mode will dominate. The phasing of the modes relative to the steady loading noise is determined primarily by $\exp \left[\mathrm{ik}\left(\phi_{0}-\pi / 2\right)\right]$ and the phase of $\mathrm{F}_{\mathrm{k}}$. For angle of attack, only the $\mathrm{k}=0$ (steady loading) and the $\mathrm{k}=1$ modes are nonzero. For positive angle of attack, the $k=1$ modes have a phase of $180^{\circ}$ for a counter-clockwise rotating propeller. That is, the unsteady loading adds to the steady loading at $\phi_{0}=180^{\circ}$ and subtracts from it at $\phi_{0}=0^{\circ}$. The noise however, lags the loading by $90^{\circ}$ for the dominant $k=-1$ mode and peaks $90^{\circ}$ later. Thus for an aircraft with its nose up relative to the flight path (i.e., positive angle of attack) the noise will peak below the aircraft and is minimum above it. At the sides, the noise due to unsteady loading is out of phase with the steady loading noise and increases equally on both the right and left sides but not as much as below the propeller.

For clockwise rotating propellers, the phase of the loading changes by $180^{\circ}$, but the sign of $J_{-m B+1}$, which now dominates, is the opposite of that of $J_{-m B}$ and the two effects cancel. Thus, the above description of the circumferential noise variation due to unsteady loading applies independent of the direction of blade rotation.

The axial source motion, due to angle of attack, is responsible for the Bessel function $\mathrm{J}_{\mathrm{n}_{2}}\left(\mathrm{z}_{2}\right)$. The argument, $\mathrm{z}_{2}$, is proportional to $\beta$, the angle of attack, and, to a first order of approximation, the magnitude of $J_{n_{2}}\left(z_{2}\right)$ is proportional to $\beta^{n_{2}}$. However, the value of $z_{2}$ is not small at angles away from the plane of rotation, and the first order approximation cannot be used. Further, orders of $J_{n_{2}}$ that decrease the order of $J_{ \pm m B}+k+n_{2}$ increase the magnitude of $J_{ \pm m B}+k+n_{2}\left(z_{1}\right)$ relative to those that increase the order. Thus it is not obvious how the relative amplitudes of the modes will compare. To facilitate this comparison relative modal amplitudes, normalized to the zero angle of attack amplitude, are listed in table I for typical conditions. As can be seen in table I, the magnitude of the $n=1$ mode is small compared to the other modes. At far forward and aft angles, i.e., $30^{\circ}$ and $150^{\circ}$ several modes are important, with the $\mathrm{n}=-1,-2,-3$ modes having a greater amplitude than the steady loading. It is interesting to note that the $n=0$ mode is less than 1 away from the propeller plane i.e., angle of attack has modified the nonspinning mode. This is a higher order effect, since the lowest power of $z_{2}$ in $\mathrm{J}_{0}\left(z_{2}\right)$ is 2 .

The angle of attack effect on force direction manifests itself in the parenthesis following $\sin \alpha$ in the fourth line of equation (18). This term, unlike the effect due to axial source motion, has maximum effect on radiation in the propeller plane and it has no effect at circumferential angles of $0^{\circ}$ and $180^{\circ}$.

\section{Comparison Wi th Data}

The results presented in this report are derived for a source moving with Mach number $M_{X}$ in the positive $x$ direction and a stationary observer. As pointed out by, Morse and Ingard, (ref. 15), this is equivalent to a stationary source with the fluid moving with Mach number $M_{X}$ in the negative $x$ direction, except that with the observer fixed relative to the source, there is no Doppler 
shift in frequency. Thus for comparison with wind tunnel data, equations (18) and (26) are evaluated with no Doppler shift in frequency.

Results of the analysis are compared with the data of reference 1 for the single rotation SR-7 propeller and the data of reference 2 for the F7A7 counterrotation propeller. For the SR-7 tests, the data were obtained at fixed microphone locations that did not vary with propeller angle of attack. This is consistant with the coordinate system of the present analysis which is fixed relative to the flow direction and not the propeller axis. A picture of the SR-7A propeller installed in the 9- by 15 -foot wind tunnel is shown in figure 4. (For the data used in this report, the wing was not present.) For the CR data, the microphone was on a boom that was attached to the propeller model. A sketch of this arrangement is shown in figure 5. Thus, the microphone location was fixed relative to the propeller coordinates. This is the coordinate system chosen by Mani in his analysis. However the present analysis can easily be adapted to either coordinate system by computing the noise levels at the proper observer location. In all cases the predictions and the data are compared on the basis of difference between the SPL with the propeller at angle of attack and the SPL without angle of attack. For the CR case this meant computing the noise with angle of attack at one observer location and the noise without angle of attack at another observer location, with the difference in location being due to the movement of the microphone which was attached to the propeller model.

In figure 6, predicted and measured effect of angle of attack on the noise directly below the SR-7 propeller, $\phi_{0}=270^{\circ}$, are compared. The data show a nearly linear increase in level with angle of attack with a slope of about $0.7 \mathrm{~dB}$ per degree of angle of attack. Two predicted curves are shown. For the dashed curve, the difference between predicted SPL, with angle of attack, and predicted SPL, without angle of attack, is due only to the effects of unsteady loading and unsteady thickness. (Unsteady thickness is not a major contributor.) The solid curve includes differences due to both unsteady sources and angle of attack on source location and thrust direction. As can be seen, including these added effects significantly improves the agreement between data and theory.

In figure 7 , the predicted axial variation in the effect of angle of attack is compared with the data of reference 1 for the SR-7 propeller at $10^{\circ}$ angle of attack. The comparison is made at an azimuthal angle that correspond to being below an aircraft in flight. Two predicted curves are shown. One is for modes with orders less than or equal to 1 in magnitude and the other for modes with orders up to 5 in magnitude, i.e., the limits on the $k$ and $n$ sums are -1 to +1 and -5 to +5 respectively. Including modes $w i$ th orders less than or equal to 1 is an attempt to simulate limiting the angle of attack to small values as was done by Mani (ref. 9). The simulation is not exact since the $\mathrm{n}=0$ and $\mathrm{n}= \pm 1$ modes include some higher order effects. Very little difference between the curves exists in the range of angles between $70^{\circ}$ and $110^{\circ}$ and in this region, excellent agreement exists between data and theory. At the far forward and aft angles, the two curves differ; however, the difference in the prediction obtained by increasing the number of modes does not improve the agreement with the data. Woodward (ref. 1) discussed the lobed nature of the propeller noise directivity and speculated that the secondary lobes at the far aft and forward angles may be due to reflections from the 
model support strut. It is this lobed pattern that gives rise to the variations in data at far forward and aft angles in figure 7 .

In figure 8 , the predicted effect of angle of attack on circumferential directivity of the front rotor blade passage frequency tone of the F7A7 counter-rotating propeller is compared with data of Woodward (ref. 2) as presented by Mani (ref. 16). In this comparison, the prediction procedure was modified to account for the variation of measurement location, relative to the analysis coordinate system. Again two curves are shown, one for low order modes and the other for mode orders up to \pm 5 . Again, the low mode order curve does not exactly simulate Mani's analysis since some higher order effects are included both in the basic equations and, implicitly, in the adjustments made to account for variation in observer location with angle of attack.

At $67^{\circ}$ (fig. 8(a)) significant difference exists between the curves, and the inclusion of higher order modes improves the agreement between data and theory. At $104^{\circ}$ (fig. 8(b)) no difference exists between the two curves and the theory underpredicts the effect of angle of attack by about $2 \mathrm{~dB}$. In addition, the location of the maximum and minimum are shifted. The theory predicts a minimum at $90^{\circ}$ and a maximum at $270^{\circ}$. The data are shifted from these by about $30^{\circ}$. Inclusion of a phase lag in the unsteady loading, using a flat plate response analysis, does not change the predicted levels significantly. At $129^{\circ}$ (fig. $8(\mathrm{c})$ ) the inclusion of more modes again changes the predicted levels, but does not significantly improve the agreement with the data. This is similar to the effect observed with the SR-7 propeller at forward and aft angles.

\section{CONCLUDING REMARKS}

An analysis method is presented which predicts the effect of angle $c^{c}$ attack on propeller noise. Three effects of angle of attack are identif 1 ed: (1) Variation in the magnitude of the blade loading (This effect is the one that is usually included in angle of attack analyses.) (2) Axial source motion, and (3) Variation in the direction of blade loading. The analysis removed the restriction of small angle of attack, that was assumed in a previous analysis. It is shown that the inclusion of the effects of angle of attack on source motion and loading direction significantly improve agreement with data compared to analyses that only include unsteady loading effects. It is also shown that including higher order effects of angle of at tack in the analysis changes the predicted noise at forward and aft angles but has little effect near the propeller plane. 


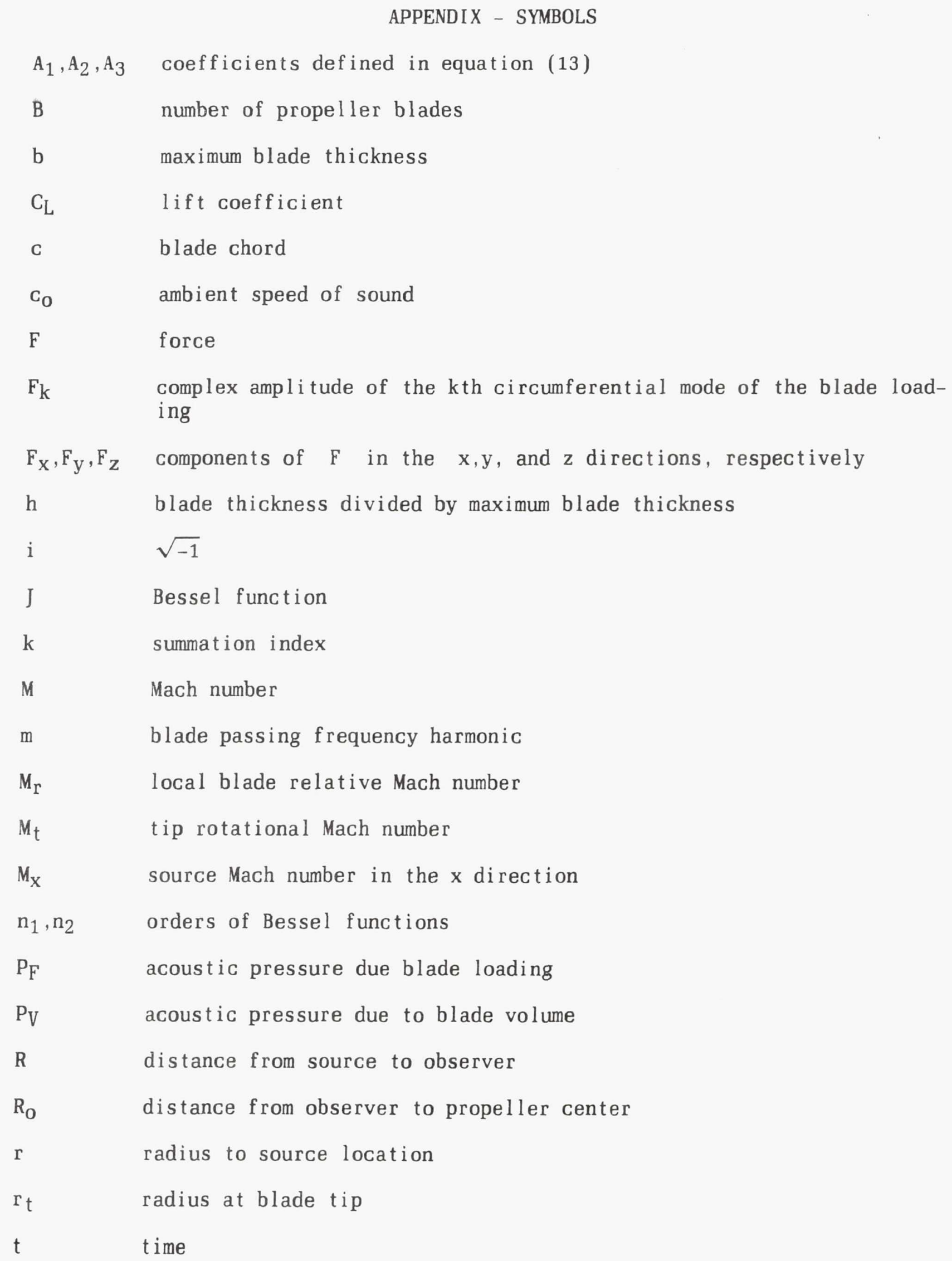




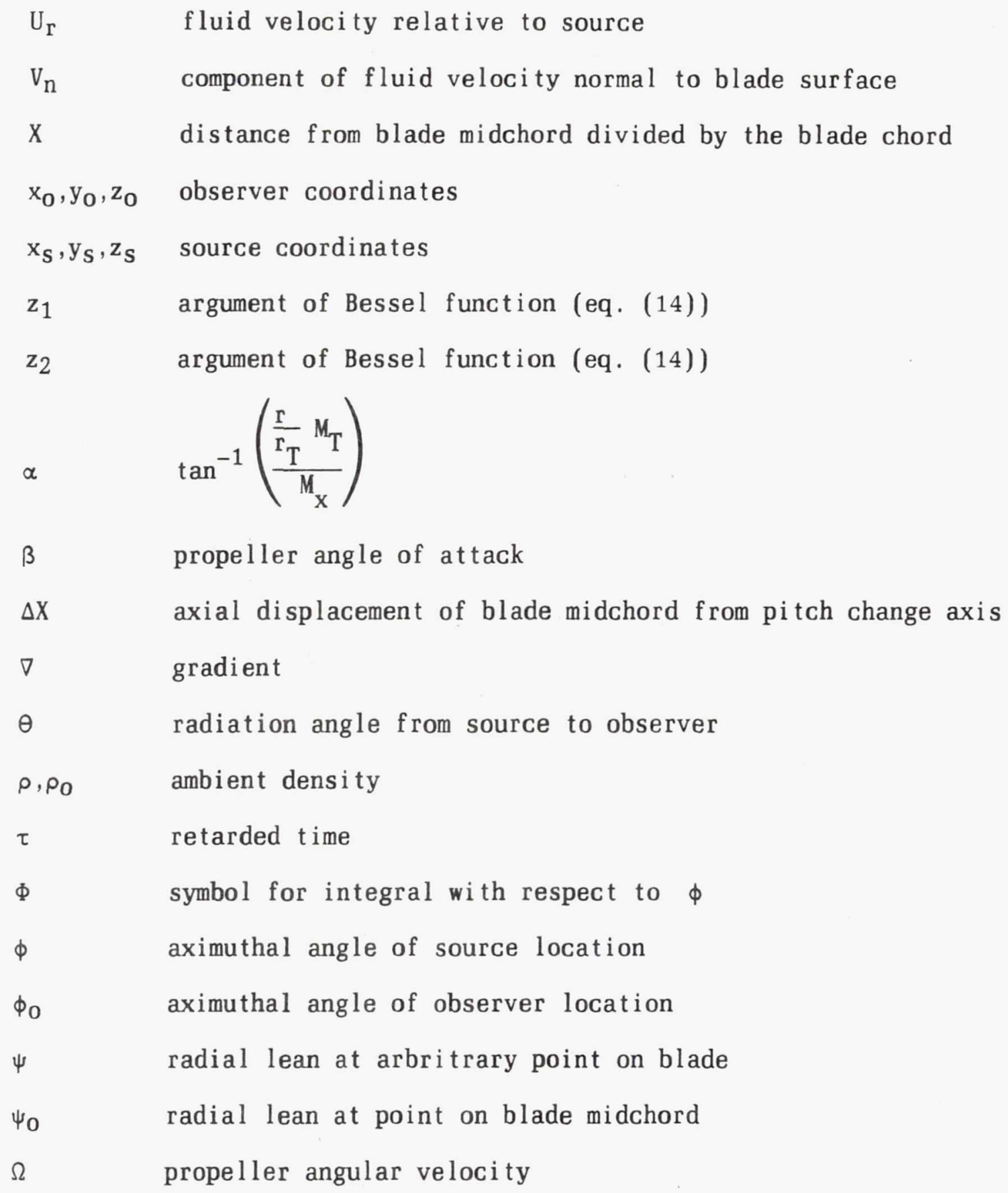




\section{REFERENCES}

1. Woodward, R.P.: Measured Noise of a Scale Model High Speed Propeller at Simulated Takeoff/Approach Conditions. AIAA 87-0526, Jan. 1987. (Also, NASA TM-88920).

2. Woodward, R.P.: Noise of a Model High Speed Counterro tation Propeller at Simulated Takeoff/Approach Conditions (F7/A7). AIAA Paper 87-2657, 0ct. 1987 (Also, NASA TM-100206).

3. Woodward, R.P.; and Gordon, E.B.: Noise of a Model Counterrotation Propeller With Reduced Af $t$ Rotor Diameter at Simulated Takeoff/Approach Conditions (F7/A3). AIAA Paper 88-0263, Jan. 1988 (Also, NASA TM-100254).

4. Block, P.J.W.: The Effects of Installation On Single- and Counter-Rotation Propeller Noise. AIAA Paper 84-2263 0ct. 1984.

5. Block, P.J.W.: Experimental Study of the Effects of Installation on Single- and Counter-Rotation Propeller Noise. NASA TP-2541, 1986.

6. Morse, P.M.; and Ingard, K.U.: Theoretical Acoustics. McGraw-Hill, 1968, pp. $740-747$.

7. Goldstein, M.E.: Aeroacoustics, McGraw-Hill, 1976, pp. 163-171.

8. Hanson, D.B.: Noise of Counter-Rotation Propellers. J. Aircraft, vol. 22, no. 7 , July 1985, pp. 609-617.

9. Whitfield, C.E., et al.: High Speed Turboprop Aeroacoustic Study (Single Rotation), Vol. 1, NASA CR-182257-VOL-1 Model Development, 1989.

10. Mani, R.: The Radiation of Sound From a Propeller at Angle of Attack. NASA CR-4264, 1990.

11. Stuff, R.: Noise Field of a Propeller with Angular Inflow. AIAA J., vol. 26, no. 7, July 1988, pp. 777-782.

12. Padula, S.L.; and Block, P.J.W.: Predicted Changes in Advanced Turboprop Noise with Shaft Angle of Attack. J. Propulsion Power, vol. 1, no. 5, Sept.-0ct. 1985, pp. 381-387.

13. Parry, A.B.; and Crighton, D.G.: Theoretical Prediction of Single Rotation Propeller Noise. AIAA Paper 86-1891, July 1986.

14. Abramowitz, M.; and Stegun, I.A.: Handbook of Mathematical Functions with Formulas, Graphs, and Mathematical Tables. National Bureau of Standards Applied Mathematics Series 55, 1964, p. 361.

15. Morse, P.M.; and Ingard, K.U.: Theoretical Acoustics. McGraw-Hill, 1968, p. 735 .

16. Whitfield, C.E.; Mani, R.; and Gliebe, P.R.: High Speed Turboprop Aeroacoustic Study (Counterrotation) Volume I - Model Development. NASA CR-185241, 1990. 
TABLE I . - COMPARISON OF RELATIVE MODEL AMPLITUDES

Value of $\frac{J_{m B+n_{2}}\left(z_{1}\right) J_{n_{2}}\left(z_{2}\right) \exp \left[i n_{2}\left(\phi_{2}-\frac{\pi}{2}\right)\right]}{J_{m B}\left(z_{1}\right)}$ with $m B=8, M_{t}=0.8 ; M_{x}=0.2$;

angle of attack $=10^{\circ}$.

(a) Azimuthal angle $=90^{\circ}\left(\phi_{0}=\pi / 2\right)$

\begin{tabular}{|r|r|r|l|r|r|}
\hline $\mathrm{n}$ & \multicolumn{5}{|c|}{$\theta$} \\
\cline { 2 - 6 } & \multicolumn{1}{|c|}{30} & \multicolumn{1}{|c|}{60} & \multicolumn{1}{c|}{90} & 120 & \multicolumn{1}{c|}{150} \\
\hline 1 & 0.08 & 0.08 & 0.08 & -0.03 & -0.03 \\
0 & .77 & .92 & 1 & .98 & .91 \\
-1 & -2.16 & -.81 & .11 & .55 & -2.14 \\
-2 & 2.38 & .29 & .01 & .15 & 2.12 \\
-3 & -1.45 & -.06 & 0 & .02 & -1.18 \\
-4 & .53 & 0 & 0 & 0 & .40 \\
-5 & -.12 & 0 & 0 & 0 & .08 \\
\hline
\end{tabular}

(b) Azimuthal angle $=270^{\circ}\left(\phi_{0}=3 \pi / 2\right)$

\begin{tabular}{|r|r|r|l|r|r|}
\hline \multirow{2}{*}{$\mathrm{n}$} & \multicolumn{5}{|c|}{$\theta$} \\
\cline { 2 - 6 } & 30 & 60 & \multicolumn{1}{|c|}{90} & 120 & \multicolumn{1}{c|}{150} \\
\hline 1 & -0.08 & -0.06 & 0.01 & 0.05 & 0.04 \\
0 & .81 & .96 & 1 & .95 & .89 \\
-1 & 2.01 & .61 & -.11 & -.85 & -2.33 \\
-2 & 1.99 & .16 & .01 & .37 & 2.54 \\
-3 & 1.09 & .02 & 0 & -.07 & -1.55 \\
-4 & .36 & 0 & 0 & 0 & .58 \\
-5 & .07 & 0 & 0 & 0 & -.14 \\
\hline
\end{tabular}

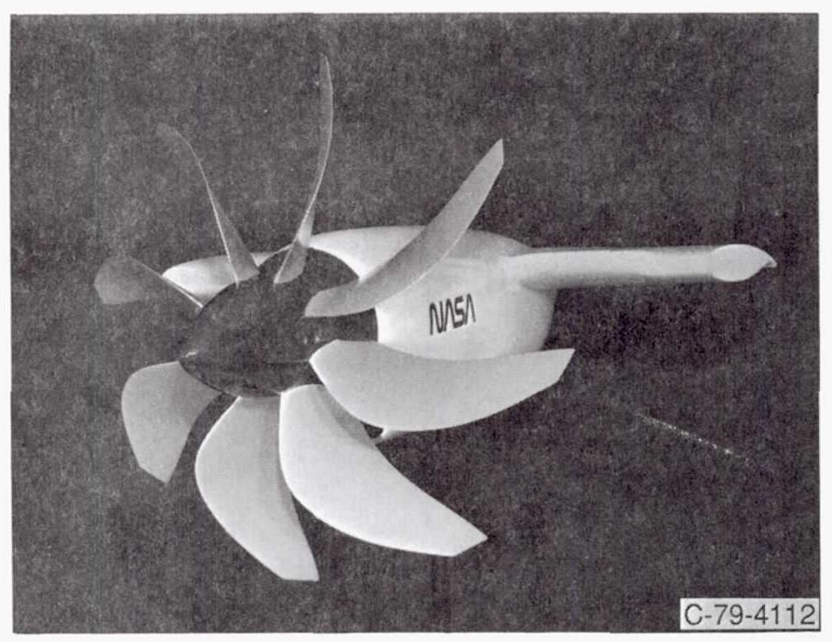

Figure. 1-Model of advanced propeller. 


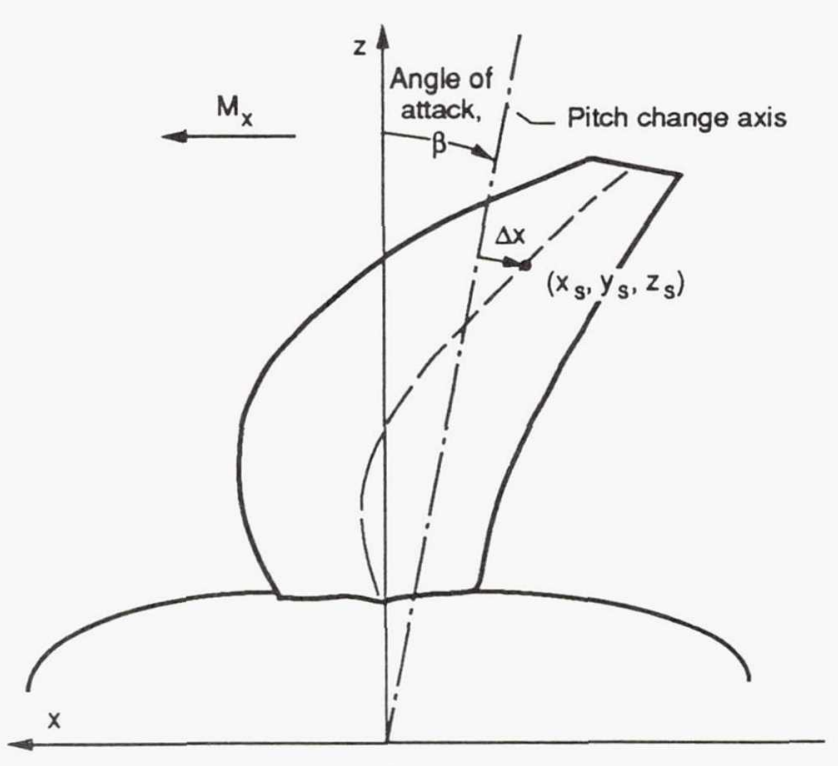

(a) Side view.

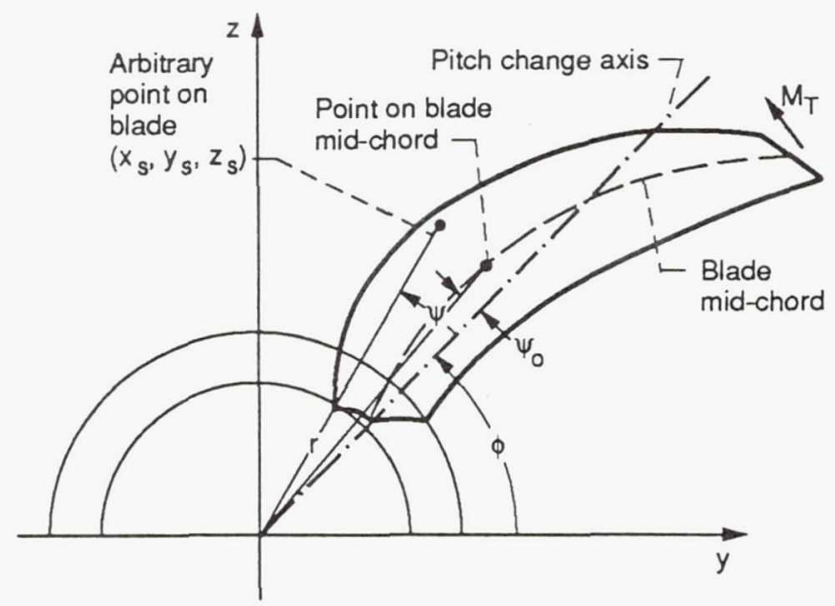

(b) View, forward looking aft.

Figure. 2- Propeller blade coordinates.

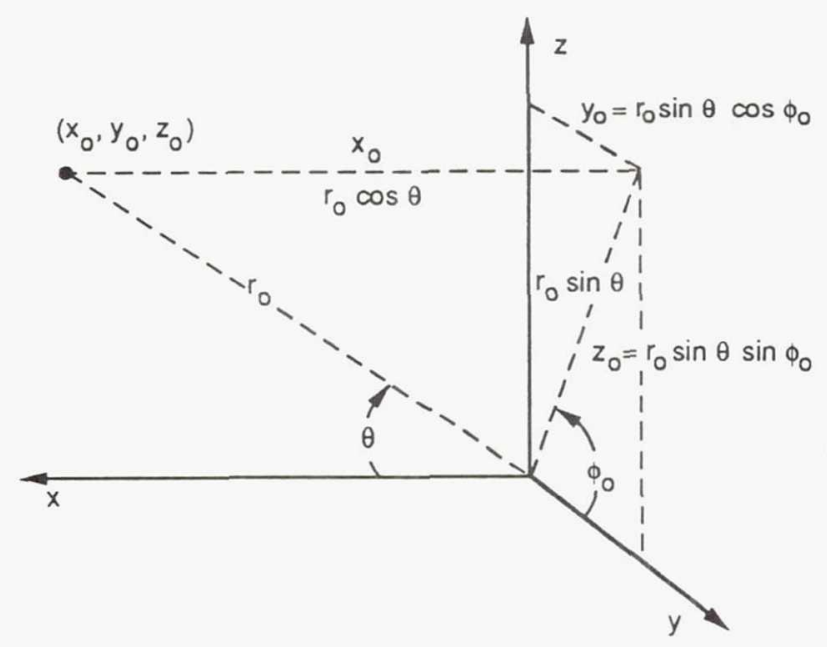

Figure. 3- Observer coordinates.

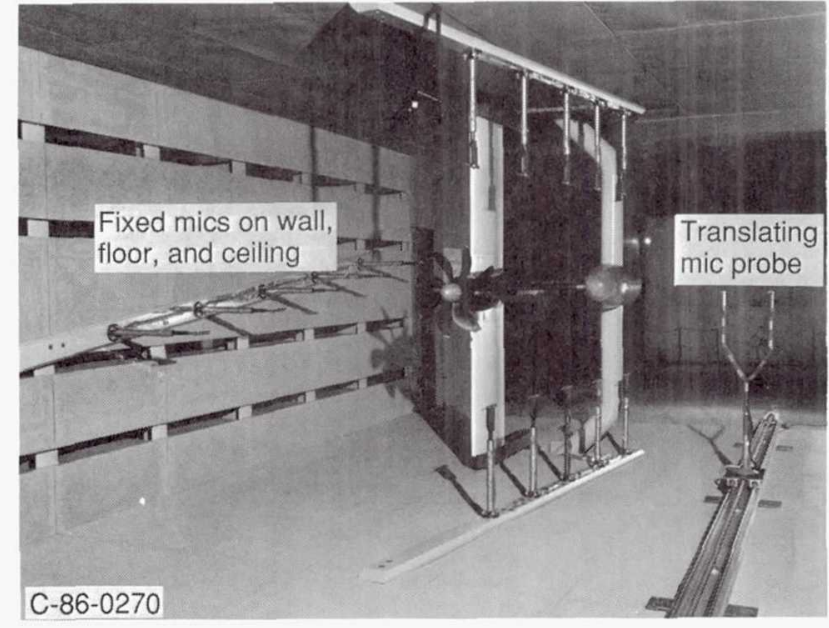

Figure. 4-Photo of SR-7A turboprop in the $9 \times 15$ anechoic wind tunnel. 


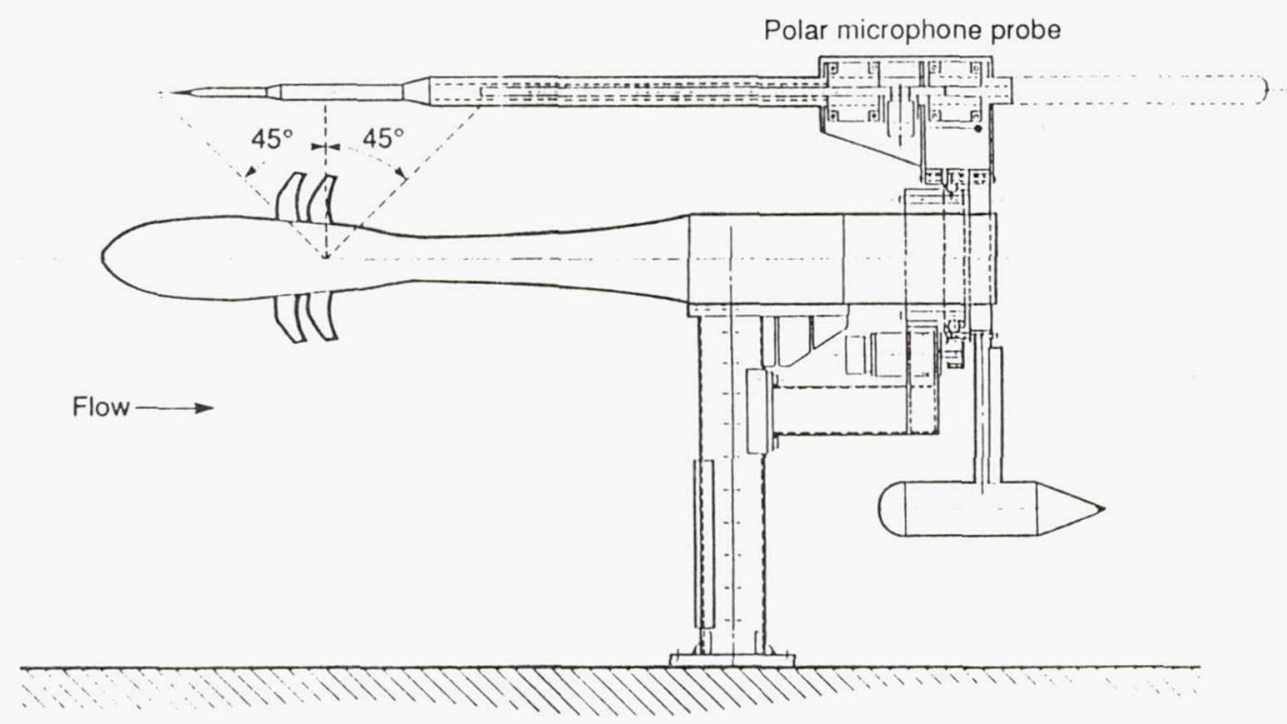

Figure. 5- Sketch of the counter-rotation turboprop model and polar microphone probe.

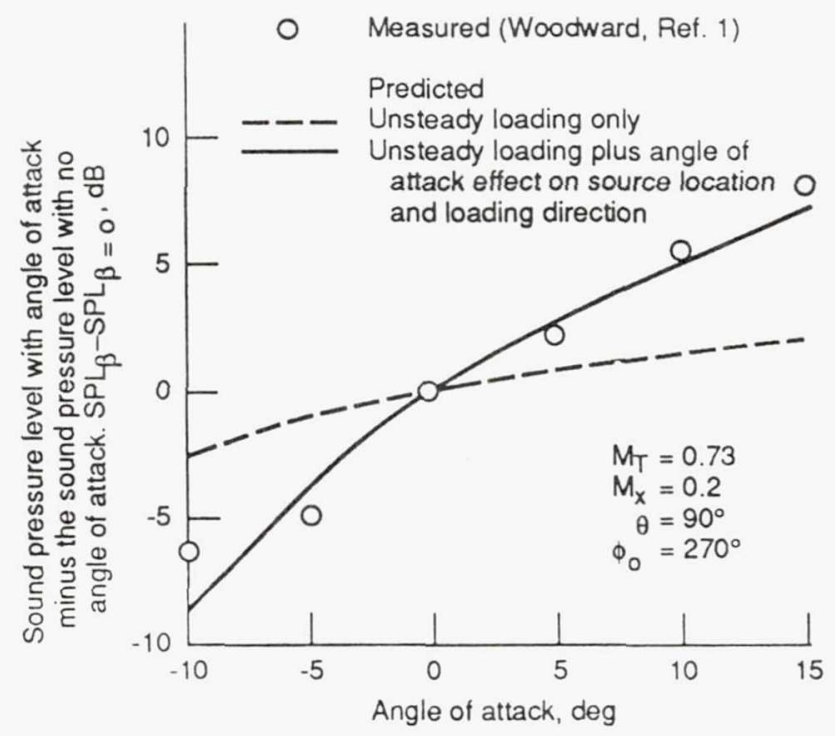

Figure. 6-Comparison of predicted and measured effect of angle of attack on the blade passing tone sound pressure level for the SR-7.

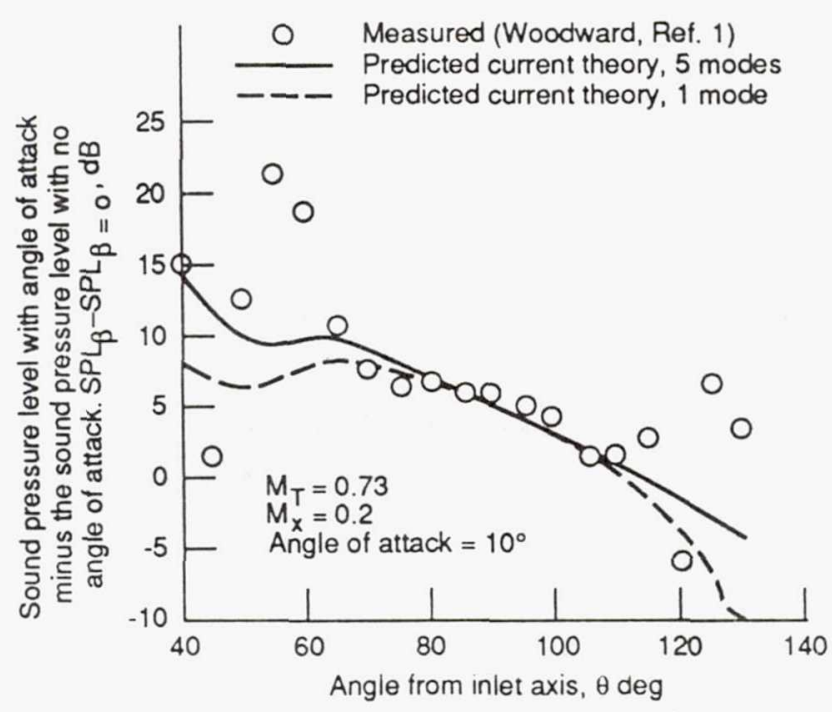

Figure. 7-Comparison of predicted and measured effect of angle of attack on the axial directivity of the SR-7 blade passing tone below the propeller I.e. $\phi_{0}=270$. 


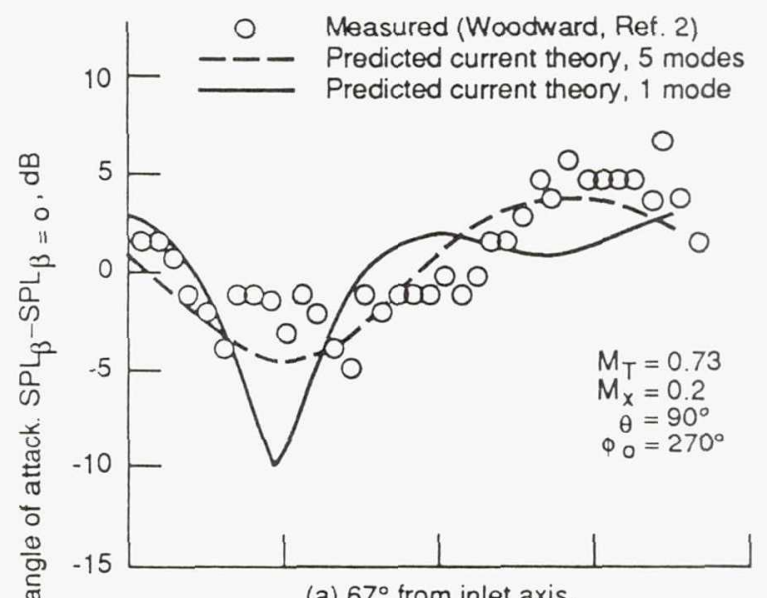

(a) $67^{\circ}$ from inlet axis

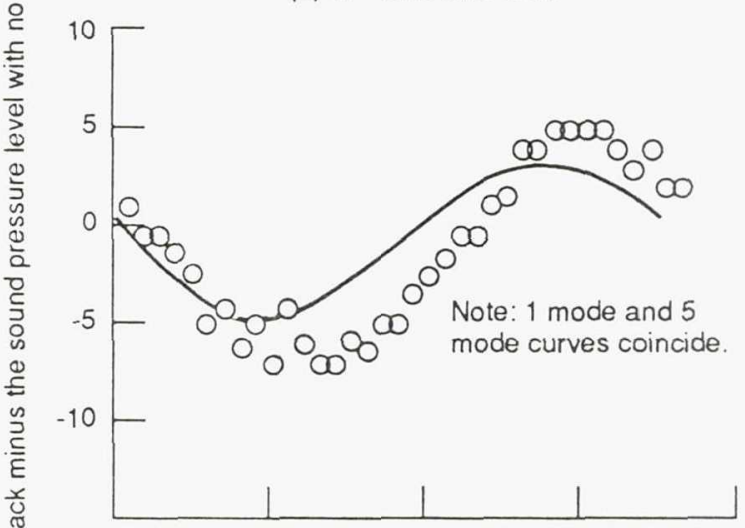

(b) $104^{\circ}$ from inlet axis.

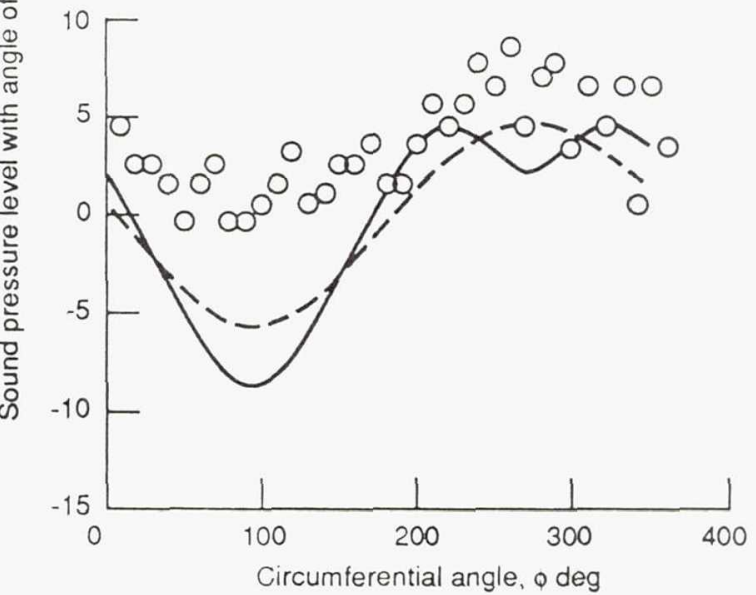

(c) $129^{\circ}$ from inlet axis.

Figure. 8-Comparison of predicted and measured effect of angle of attack on the circumferential directivity of the F7A7 front rotor blade passing tone. Angle of attack $=8$ degrees 


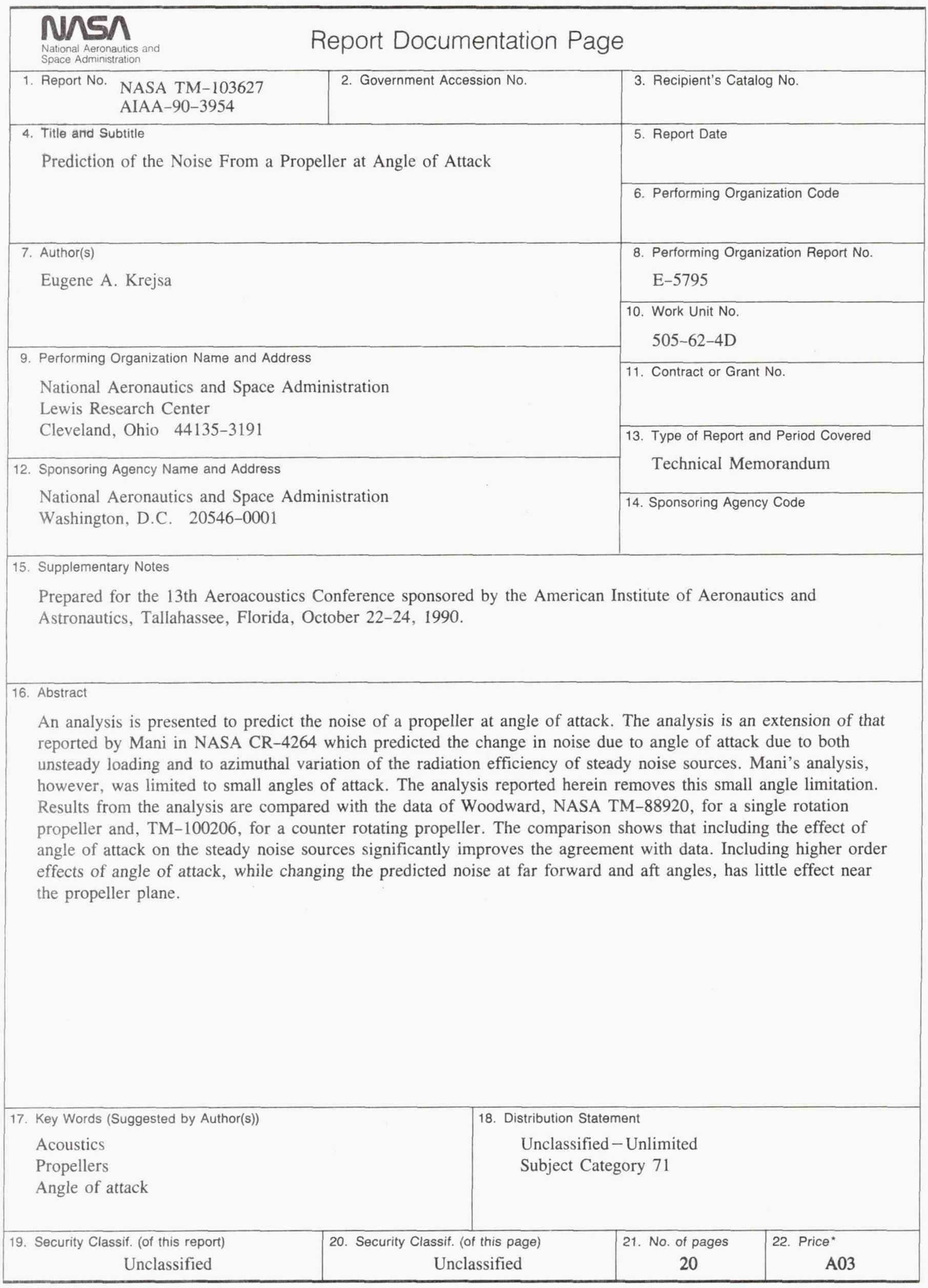

